\title{
Impedance Coordinative Control for Cascaded Converter in Bidirectional Application
}

\author{
Yanjun Tian ${ }^{1}$, Fujin Deng ${ }^{1}$, Zhe Chen $^{1}$, Xiaofeng Sun $^{2}$, Yanting $\mathrm{Hu}^{3}$ \\ ${ }^{1}$ Aalborg University, Denmark \\ ${ }^{2}$ Yanshan University, China \\ ${ }^{3}$ University of Chester, United Kingdom
}

\begin{abstract}
Two stage cascaded converters are widely used in bidirectional applications, but the negative impedance may cause system instability. Actually the impedance interaction is much different between forward power flow and reversed power flow, which will introduce more uncertainty to the system stability. This paper proposes a control method for the constant power controlled converter in cascaded system, and consequently it can change the negative impedance of constant power converter into resistive impedance, which will improve the cascaded system stability, as well as merge the impedance difference between forward and reversed power flow. This paper addresses the analysis with the topology of cascaded dualactive-bridge converter (DAB) with inverter, and the proposed control method can also be implemented in unidirectional applications and other general cascaded converter system. The effectiveness has been validated by both simulation and experimental results.
\end{abstract}

Index Terms-- Bidirectional control, cascaded converter, impedance interaction, impedance control, system stability.

\section{INTRODUCTION}

With the increasing of renewable energy based power generations, the power electronics converters are widely used as the flexible and efficient interface component for connecting renewable energy systems with the utility grid, as well as local loads [1]. Among the power converters, the cascaded topologies are widely used in the PV plant, energy storage system, wind power system, motor drives and many other applications [2].

Required by higher demands on the performance of AC/DC or DC/DC converters, filters, DC buses, complex loads, the design of power system is constantly increasing [3]. In 1979, Middlebrook analyzed that for the cascaded system, the stability not only relies on the well designing of individual converter, but also the impedance interaction between the cascaded sub-converters, known as the impedance interaction [4]. Gradually the design is shifting from the knowledge of controlling single device, to coordinating the connected sub-devices. So the impedance-based stability and transient-performance analyses are applied in more and more occasions [5].

One problem for cascaded converter system is the potential stability degradation, and this may occur when connecting two individual controlled switching converters in series. This phenomenon can be figured with two interpretations [6]: One is that the stability degradation is caused by the interaction among the feedback loops between the interconnected converters; the other interpretation recognizes the problem as the constant power load (CPL) effect. Under constant

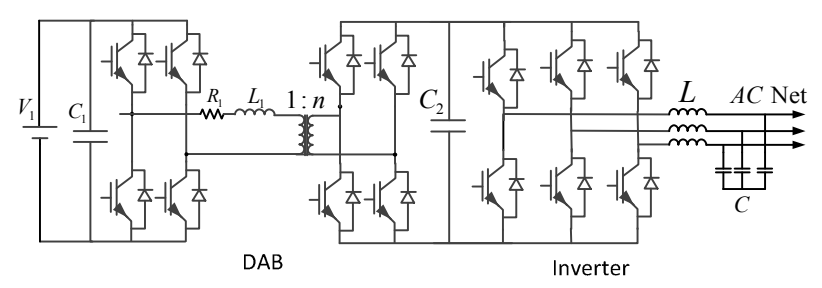

Fig. 1 Cascaded converter with two elementary converters (front-end $\mathrm{DAB}$ and rear-end three-phase inverter).

instantaneous power control, viewed from the input terminal, the converters behave as a constant power loads [7], which is called negative impedance. The negative impedance tends to destabilize the power system [8].

The stability in cascaded converter can be evaluated by the impedance interaction. If the source converter output impedance $Z_{o}$ is much less than the load converter input impedance, the stability of the cascaded system can be guaranteed, called Middlebrook criterion. Several other criterions were developed in the past few years [9]. But in bidirectional applications, when power flow reverses, the impedance of CPLs will become resistive impedance. This variation will introduce more uncertainty to the system stability.

In this paper, an impedance coordinate control method for CPLs is proposed. In the proposed control, the CPL converter behave as resistive impedance in both forward and reversed power flow, and this can not only get rid of the instability introduced by the negative impedance in conventional control, but also make the system behave unified between forward and reversed power flow.

Where galvanic isolation is required, the DC-DC converter must have a high-frequency transformer included, like the Dual-Active-Bridge (DAB) converter shown in Fig. 1, when cascaded to the voltage-source inverter. The DAB converter has been used solid-state transformers, and is generally attractive because of its high power density, bidirectional power flow ability and zero voltage switching [10]. It is therefore the chosen sub-converter for cascading with the inverter to form the DABCI converter ( $\mathrm{C}$ and $\mathrm{I}$ stand for cascaded and inverter respectively). Other sub-converters can also be cascaded without affecting control findings uncovered in the paper.

\section{IMPEDANCE INTERACTION MODELLING AND ANALYSIS}

\section{A. Impedance interaction in bidirectional applications}

To illustrate the effects of source-load converter interactions, the system can be spilt into two sub-systems: the source and the load sub system, as in Fig. 2. 


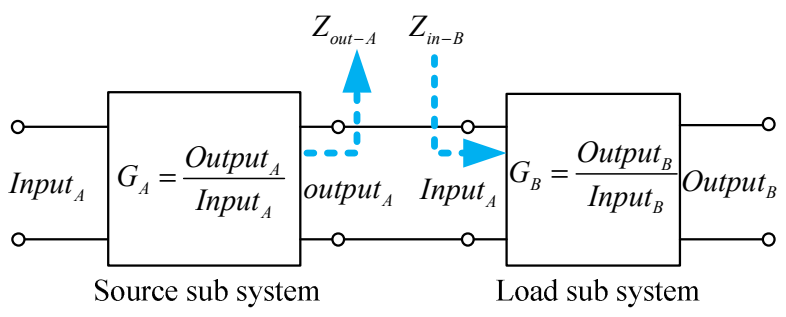

Fig. 2 Inter connection of the cascaded sub system

The overall input to output transfer function is [11]:

$$
G_{A B}=\frac{\text { output }_{B}}{\text { Input }_{A}}=G_{A} G_{B} \frac{1}{1+T_{m}}
$$

where $G_{A B}$ is the overall input to output transfer function; $G_{A}$ is the input to output transfer function of source sub system. $G_{B}$ is the input to output transfer function of load sub system. $T_{m}$ is the impedance interaction item, defined as the minor loop gain[12].

If the source sub system is voltage fed converter, which means the source converter controls the output voltage, then the minor loop gain $T_{m}$ is defined as[13]:

$$
T_{m}=\frac{Z_{\text {out }-A}}{Z_{\text {in }-B}}
$$

where $Z_{\text {out }-A}$ is the output impedance of sub source system; $Z_{i n-B}$ is the input impedance of sub load system.

If the source sub system is the current fed converter, which means the output A is current controlled, then the minor loop gain $T_{m}$ becomes as[14]:

$$
T_{m}=\frac{Z_{\text {in-B }}}{Z_{\text {out }-A}}
$$

So in the minor loop gain $T_{m}$, the numerator has to be the internal impedance of the subsystem containing the voltage source or load, and the denominator impedance have to be the internal impedance of the subsystem containing the current load or source, respectively.

Returning to Fig. 1, between the two sub-converters is a DC-link capacitor $C_{2}$ for smoothening voltage ripple in the steady state [15], which otherwise, may affect stability of the cascaded converter [16].

Conventionally for the voltage $V_{d c}$ across $C_{2}$ to be regulated by a controller through one of the subconverters $[17,18]$. The other sub-converter will then control power flow through the overall cascaded converter.

\section{B. DAB impedance modelling}

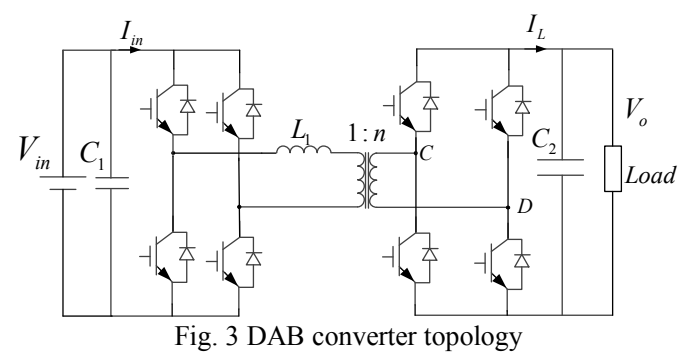

DAB converter consists of two H-Bridges and a high frequency transformer, as shown in Fig. 3. The transferred power can be controlled by the phase shift between the two H-Bridges, and the average current of $\mathrm{DAB}$ converter can be derived as:

$$
I_{L}=\frac{V_{\text {in }}}{2 n f_{s} L_{1}} D(1-\mathrm{D})
$$

where $n$ is the transformer turns ratio between secondary side and the primary side. $f_{s}$ is the switching frequency. $D=\frac{\phi}{\pi}$ is the duty ratio of the phase shift. $V_{\text {in }}$ and $V_{o}$ are respectively the input and output voltage of DAB converter. $L_{1}$ is the leakage inductance of the high frequency transformer.

So DAB converter is a current source converter [19], then the DAB voltage control loop can be designed with inner average current loop [20].Fig. 4 shows the voltage control diagram. In the voltage controller, $G$ is the gain, and it will be used to illustrate the relationship between the DAB output impedance with this controller gain.

According to Fig. 4, the DAB output impedance can be expressed as:

$$
Z_{\text {out }-D A B}=-\frac{V_{o}}{I_{o}}=\frac{s}{C s^{2}+\left(K_{V P} s+K_{V i}\right) G \cdot G_{I-D A B}}
$$

where $G_{I-D A B}$ is the closed loop transfer function of current loop, which can be obtained by Fig. 4. The bode plots of DAB output impedance can be shown in Fig. 5.

As in Fig. 5, DAB output impedance is inductive at low frequency range and capacitive at high frequency rage. The output impedance increases with the reducing of voltage controller $G$. According to Middlebrook criterion, this increasing of output impedance will make the cascaded system more unstable, because the voltage controlling source converter becomes slower, more difficult to maintain the DC-link voltage stable.

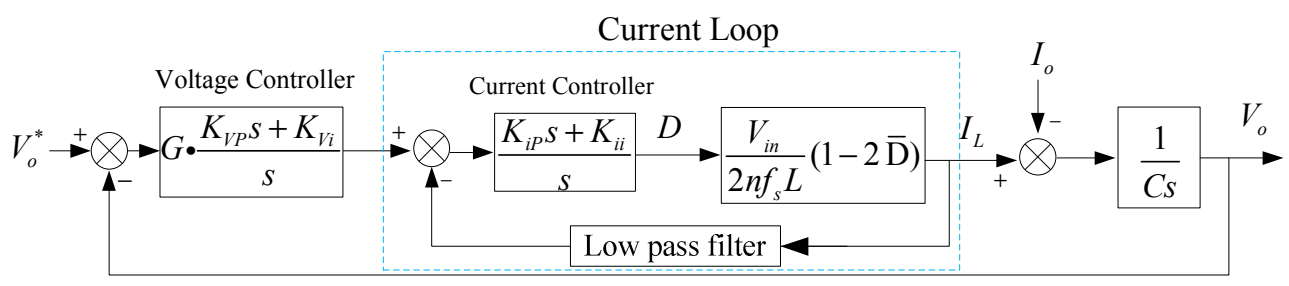

Fig. 4 Small signal model of DAB with double-loop voltage control 


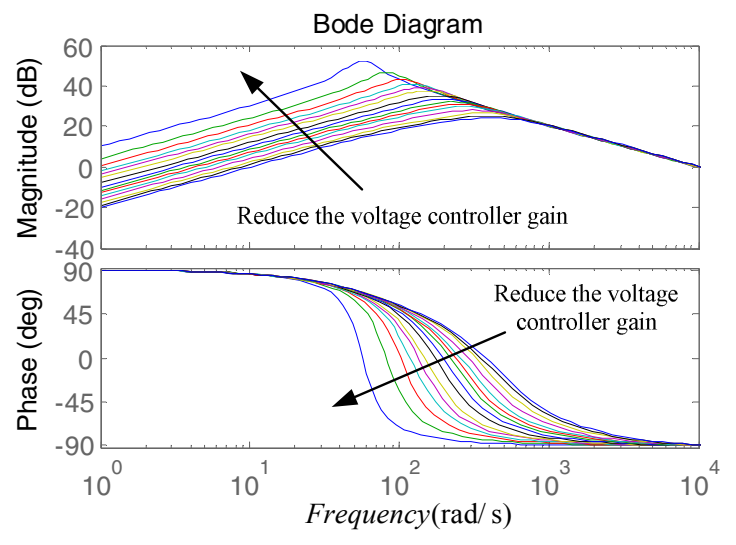

Fig. 5 Bode plots of DAB output impedance

When power flow reverses, DAB becomes the load converter, but its control diagram remains the same, then the input impedance in reversed power flow is the same as the output impedance in forward power flow.

\section{Inverter impedance modelling}

Fig. 6 shows the inverter topology and control scheme. As shown, the grid connected inverter consists of three phase half bridges with inductor filer, and $r$ is the parasitic resistance of the inductor. The control unit is the open loop direct power control [21], which is built under the synchronous rotation frame, and it is a typical constant load converter.

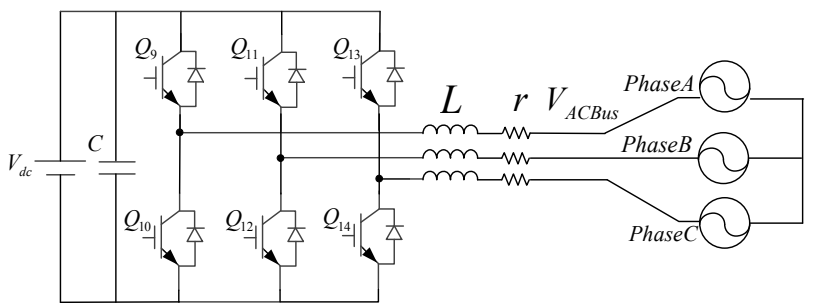

(a)Topology of grid connected converter

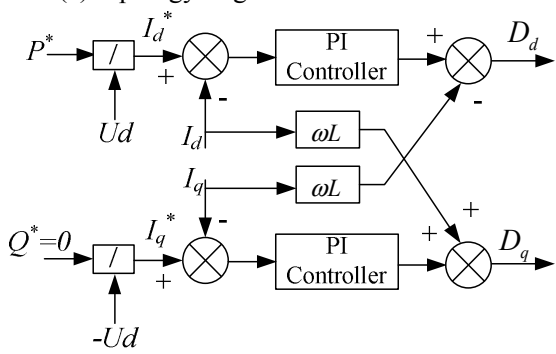

(b)Inverter control scheme

Fig. 6 Topology of grid connected inverter

The inverter input impedance in forward power flow and output impedance in reversed power flow can be expressed as (5)(6).

$$
\begin{gathered}
\overline{Z_{I N V-o}}=-\frac{\Delta V_{d c}}{\Delta I_{o}}=-\frac{V_{d c}}{1.5 \mathrm{U}_{d} \overline{G_{V_{d c} I_{d}}}-I_{o}} \\
Z_{I N V-i n}=\frac{\Delta V_{d c}}{\Delta I_{i n}}=\frac{V_{d c}}{1.5 \mathrm{U}_{d} G_{V_{d c} I_{d}}-I_{i n}}
\end{gathered}
$$

The impedance modelling detail can be found in [22].

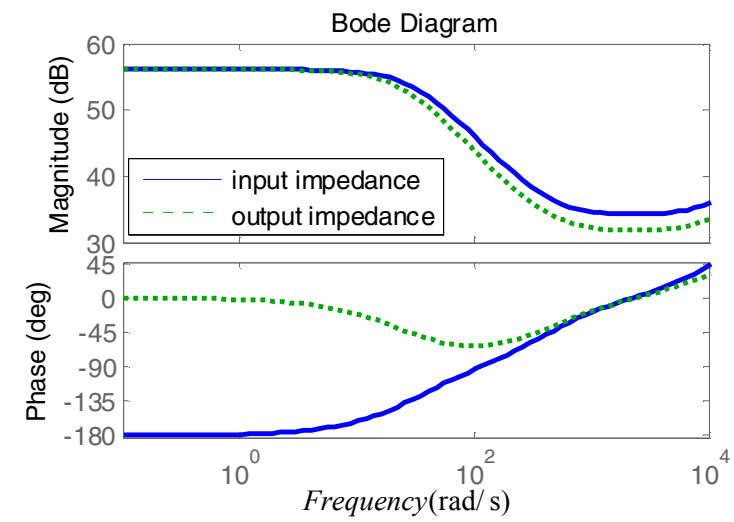

Fig. 7 Bode plots of the Input impedance of inverter with the variation of $\mathrm{K}_{\mathrm{i}}$ from 0.2 to $2 \mathrm{pu}$

The $G_{V_{d c} I_{d}}$ and $\overline{G_{V_{d c} I_{d}}}$ are respectively the transfer function from the input of DC-link voltage to the output of $\mathrm{D}$ axis current, as:

$$
\begin{aligned}
\overline{G_{V_{d c} I_{d}}} & =\frac{-D_{d} s}{L s^{2}+\left(r+V_{d c} K_{P-i n v}\right) s+V_{d c} K_{i-i n v}} \\
G_{V_{d c} I_{d}} & =\frac{D_{d} s}{L s^{2}+\left(r+V_{d c} K_{P-i n v}\right) s+V_{d c} K_{i-i n v}}
\end{aligned}
$$

Fig. 7 shows bode plots of inverter input impedance, and the rectifier mode output impedance. As shown, the inverter input impedance is negative in the low frequency range, with the phase shift of $-180^{\circ}$. With the increasing of frequency, the phase shift increases from $-180^{\circ}$ towards $90^{\circ}$. The rectifier output impedance is resistive in the low frequency range, different from the inverter input impedance in forward power flow. For the constant power converter with the same value of transmitted power, the output impedance in reversed power flow has an opposite sign with the input impedance in forward power flow.

So for the converter under constant power control, in the low frequency range, the input impedance in forward power flow is negative, but the output impedance in reversed power flow is resistive. Because of the difference in the impedance calculation, as in (5) and (6), the low frequency input impedance in forward power flow is $-\left(V_{\mathrm{dc}}^{2}\right) / P$, while the output impedance in reversed power flow is $\left(V_{\mathrm{dc}}^{2}\right) / P$.

\section{Impedance interaction in conventional control}

Based on the aforementioned impedance modelling, the minor loop gain of the impedance interaction $\left(T_{m}\right)$ can be obtained, as shown in Fig. 8. In forward power flow, the phase shift of $T_{m}$ is from $630^{\circ}$ in the low frequency range to $180^{\circ}$ in the high frequency range; in reversed power flow, the phase shift of $T_{m}$ is from $90^{\circ}$ in the low frequency range to $-155^{\circ}$ in the high frequency range. So dramatic phase shift in forward power flow indicates the stability in reversed power flow is better than forward power flow. The nyquist plots of $T_{m}$ in both forward and reversed power flow are shown in Fig. 9. 


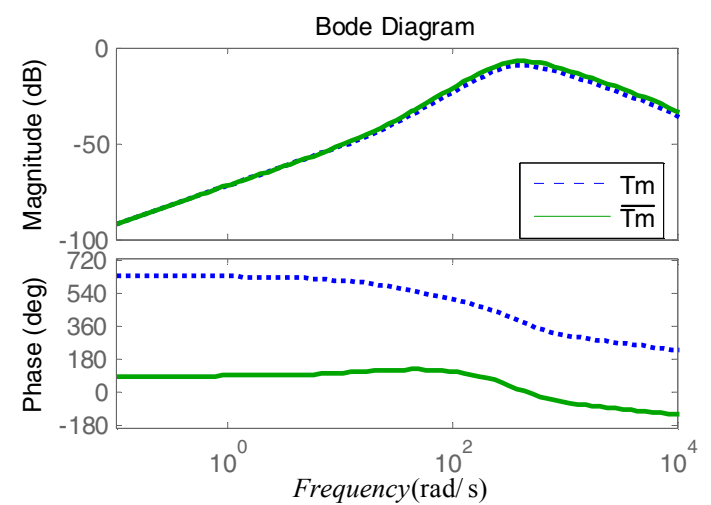

Fig. 8 Bode plots of the minor loop gain $\left(T_{m}\right)$ in bidirectional applications

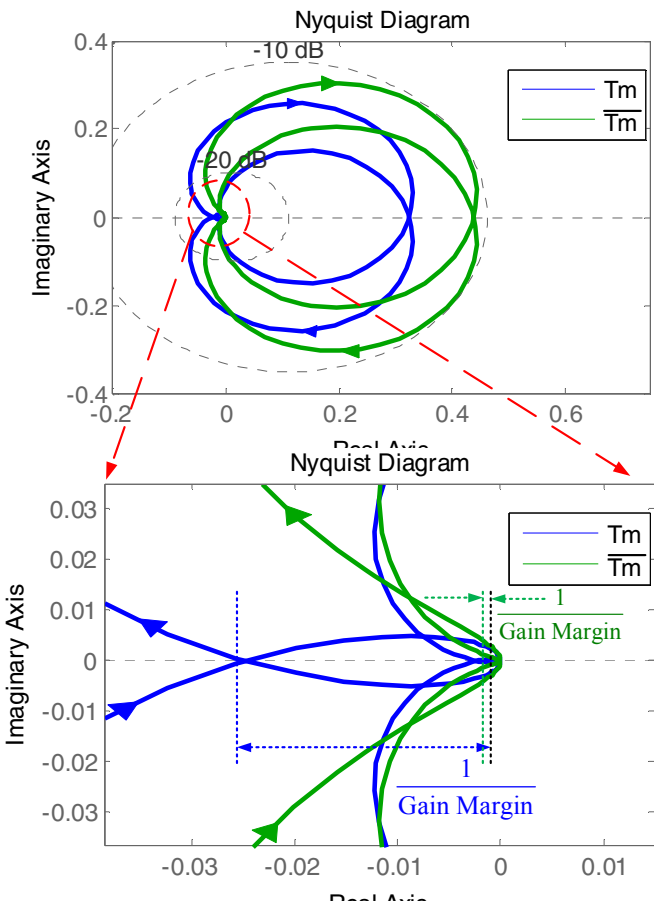

Fig. 9 Nyquist plots of the minor loop gain in bidirectional application

As shown in Fig. 9, the blue line is the nyquist plot in forward power flow, and the green line is the nyquist plot in reversed power flow. Under the same situation, the gain margin in reversed power flow is much bigger than forward power flow, which means that this cascaded system in reversed power flow is more stable than that in the forward power flow.

Without changing the control scheme, the stability difference in forward and reversed power flow may introduce more uncertainty to the cascaded system. So this paper proposed an impedance coordinate control for the cascaded converter, and it can modify the negative impedance of CPLs into resistive load, and greatly merge the stability difference in both forward and reversed power flow.

\section{IMPEDANCE COORDINATIVE CONTROL FOR THE CASCADED CONVERTER}

As previously mentioned, the impedance of voltage controlled DAB converter stay the same between forward and reversed power flow, but the impedance of constant power converter is different between forward power flow and reversed power flow. In the proposed control, the impedance of constant power converter is modified to have the similar performance between forward and reversed power flow. To change the impedance of constant load converter, an impedance controller is implemented in the power loop. The control scheme of the proposed control is shown in Fig. 10.

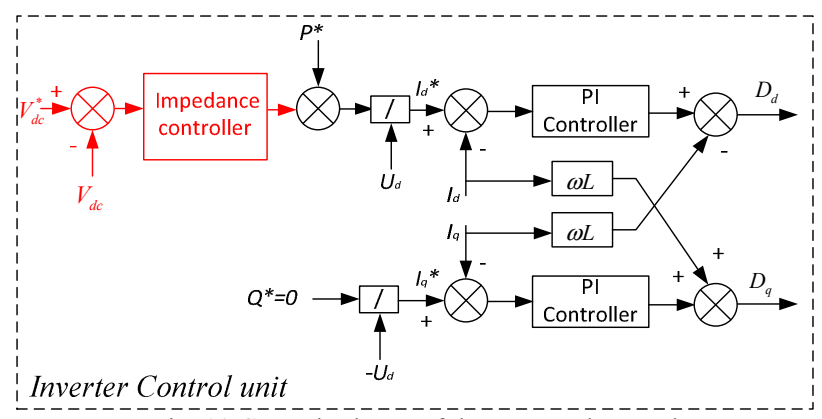

Fig. 10 Control scheme of the proposed control

\section{A. Impedance Modelling}

With the impedance controller, inverter input impedance can be obtained as:

$$
Z_{I N V-i n}=\frac{\Delta V_{d c}}{\Delta I_{i n}}=\frac{V_{d c}}{1.5 \mathrm{U}_{d} G_{V_{d c} I_{d}}^{*}-I_{i n}}
$$

where $G_{V_{d c} I_{d}}^{*}$ is the transfer function from the input of DC voltage to the output of current on the D axis in the rotation frame, and it can be obtained by(5)(6), as well as Fig. 10, and then it can be shown as Fig. 11.

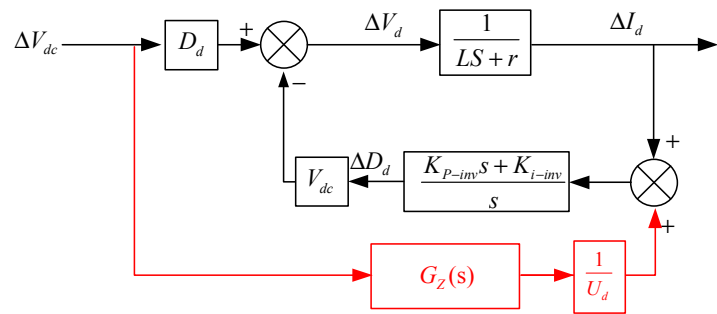

Fig. 11 Block diagram of $G_{V_{D C} I_{d}}^{*}$

$$
G_{V_{d c} I_{d}}^{*}=\frac{D_{d} s}{L s^{2}+\left(r+V_{d c} K_{P-i n v}\right) s+V_{d c} K_{i-i n v}}+\frac{G_{Z}(s)}{U_{d}}(10)
$$

where $G_{Z}(s)$ is the transfer function of the impedance controller.

As shown in (10), $G_{V_{d c} I_{d}}^{*}$ is sum of $G_{V_{d c} I_{d}}$ in conventional control and the impedance controller $G_{Z}(s) / U_{d}$. Then the input impedance in the proposed control can be obtained as (11). In reversed power flow, the output impedance in rectifier mode can be expressed as (12). 


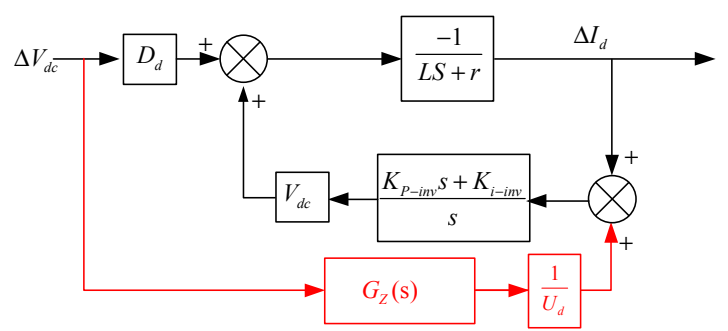

Fig. 12 Block diagram of $\overline{G_{V_{D C} I_{d}}^{*}}$

$$
\begin{gathered}
Z_{I N V-i n}^{*}=\frac{\Delta V_{d c}}{\Delta I_{i n}}=\frac{V_{d c}}{1.5 G_{Z}(s)-I_{i n}+1.5 \mathrm{U}_{d} G_{V_{d c} I_{d}}} \\
{\overline{Z_{I N V-o}}}^{*}=-\frac{\Delta V_{d c}}{\Delta I_{o}}=-\frac{V_{d c}}{1.5 \mathrm{U}_{d}{\overline{G_{V_{d c} I_{d}}}}^{*}-I_{o}}
\end{gathered}
$$

where ${\overline{G_{V_{d c} I d}}}^{*}$ is the transfer function from the input of DC-link voltage to the output of $\mathrm{D}$ axis current, as shown in Fig. 12, and it can be expressed as:

$$
\overline{G_{V_{d c} I_{d}}^{*}}=\frac{-D_{d} s}{L s^{2}+\left(r+V_{d c} K_{P-i n v}\right) s+V_{d c} K_{i-i n v}}+G_{Z}(s)
$$

Then the output impedance in rectifier mode is:

$$
{\overline{Z_{I N V-o}}}^{*}=-\frac{\Delta V_{d c}}{\Delta I_{o}}=\frac{V_{d c}}{1.5 G_{Z}(s)+I_{o}-1.5 \mathrm{U}_{d}\left(\overline{G_{V_{d c} I_{d}}}\right)}
$$

Based on (11) and (14), the consideration is to increase the input impedance and output impedance, as well as get rid of the negative impedance. Then the choice is that the dominators in (11) and (14) are both positive with small value. As in (11) and (14), if $1.5 G_{Z}(s)>I_{o}$ and $1.5 G_{Z}(s)>I_{\text {in }}$, then the negative input impedance in (12) can be avoided. To make the system impedance unaffected by the reference current, $1.5 G_{Z}(s)$ can be designed as a constant value with more than 5 times of the reference current. But the value of $G_{Z}(s)$ is not the larger the better, because arbitrarily increasing the value will introduce instability problem, and also will reduce the impedance of the inverter. So the utmost value of the impedance controller is the stability area in the inverter control block diagram of Fig. 10.

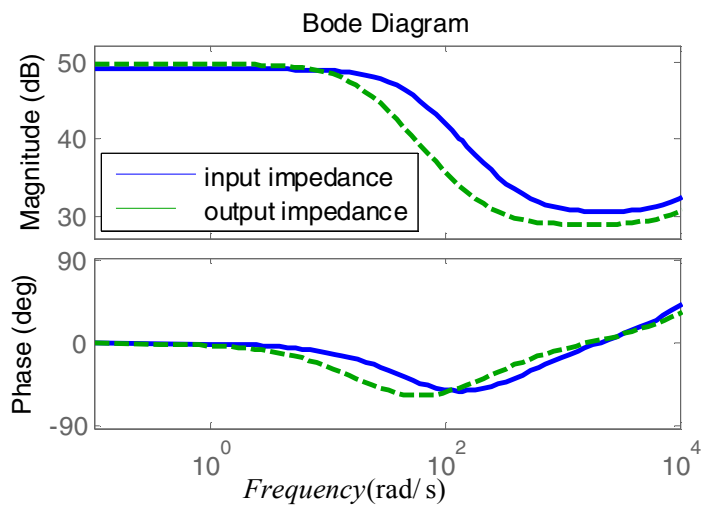

Fig. 13 Inverter Input and output impedance with the impedance controller
The input and output impedance of the proposed control is shown in Fig. 13. As shown, in the low frequency range, the input impedance in forward power is a litter larger than the output impedance in reversed power flow. But as a whole, the input impedance in forward power flow is almost the same as the output impedance in reversed power flow, so the negative impedance in conventional CPL turns to resistive impedance in the proposed control, then impedance of constant power inverter behaves almost the same in bidirectional performance.

\section{B. Analysis of minor loop gain}

Fig. 14 shows the bidirectional minor loop gain. As shown in Fig. 14, the minor loop gain in forward power flow becomes similar with reversed power flow, and the phase shift is almost the same as reversed power flow, which means the impedance interaction in the proposed control behaves the same in the both forward and reversed power flow.

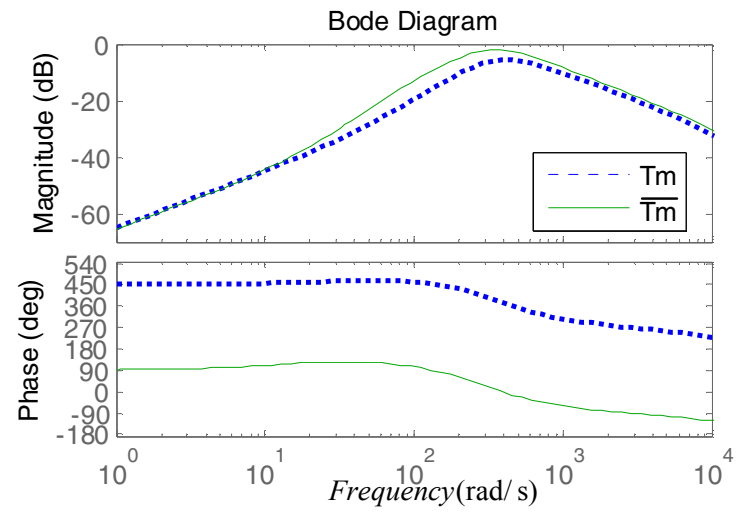

Fig. 14 Bode plots of $\mathrm{T}_{\mathrm{m}}$ in the proposed control

Fig. 15 shows the nyquist plots with the variation of DAB output impedance, and both the proposed and the conventional control are included.

Returning to Fig. 5, with the decreasing of voltage controller gain, the DAB output impedance will enlarge, and this increment will deteriorate the system stability. The nyquist plots with the variation of DAB output/input impedance are shown in Fig. 15. In Fig. 15, the input and output impedance of $\mathrm{DAB}$ are represented by $Z_{D A B}$.

In Fig. 15 (a) in the forward power flow with conventional control, the nyqusit plots are approaching the point of $(-1,0)$ with the increasing of DAB impedance, which means that the stability is tending to be unstable. In Fig. 15 (b), in reversed power flow with conventional control, with the increasing of DAB impedance, the nyquist plots show that the stability is still stable. So in conventional control, the stability in reversed power flow is more satisfied than forward power flow.

In Fig. 15 (c) and (d), with the increasing of DAB impedance, both the nyquist plots show that nyquist plot 


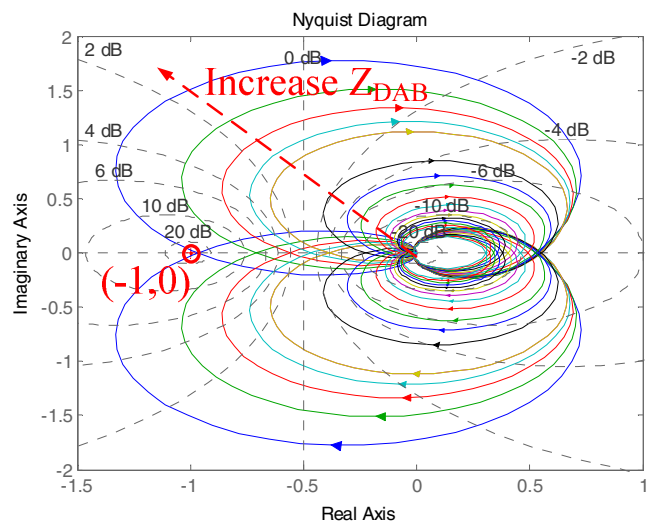

(a) $T_{m}$ in forward power flow with the conventional control

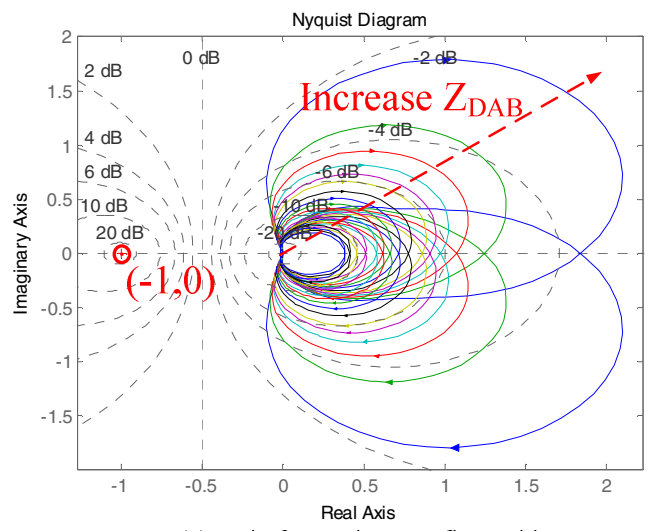

(c) $T_{m}$ in forward power flow with the proposed control

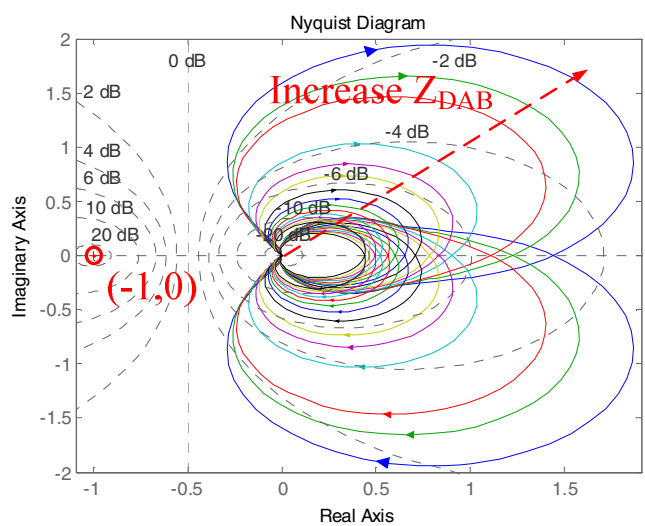

(b) $T_{m}$ in reversed power flow with the conventional control

Nyquist Diagram

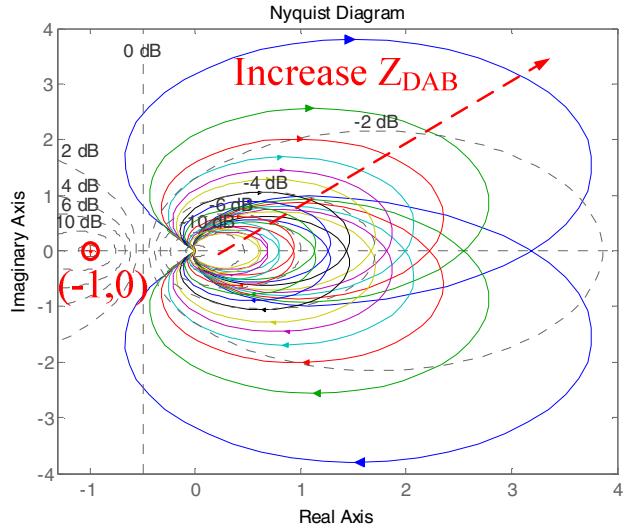

(d) $T_{m}$ in reversed power flow with the proposed control

Fig. 15 Nyquist plots with the variation of DAB output impedance

stay away from the unstable point $(-1,0)$, meaning that the system are still stable. So in the proposed control the stability in both forward and reversed power flow are both satisfactory, with similar manifestation.

\section{SIMULATION AND EXPERIMENT RESULTS}

In order to test the proposed control, a simulation model has been built in the simulation software of PLECS, and a scaled down prototype has been built in the experiment.

\section{A. Simulation results}

The simulation parameter is shown in Table1. The converter impedance can be measured by the variations of current and voltage output under certain frequency.

TABLE I

\begin{tabular}{|c|c|c|c|}
\hline SIMULATION PARAMETERS \\
\hline$V_{1}$ & $400 \mathrm{~V}$ & $L$ & $3 \mathrm{mH}$ \\
\hline$V_{2}$ & $800 \mathrm{~V}$ & $C$ & $100 \mathrm{uF}$ \\
\hline$V_{a c}$ & $311 \mathrm{~V}$ & $L_{l}$ & $0.1 \mathrm{mH}$ \\
\hline
\end{tabular}

Fig. 16 shows the measured inverter input impedance in forward power flow and the rectifier output impedance in reversed power flow. As shown, the output impedance is a litter smaller than the input impedance in forward power flow, and it is the same as the calculated results in Fig. 13. Both the input and output impedance are resistive in the low frequency range.

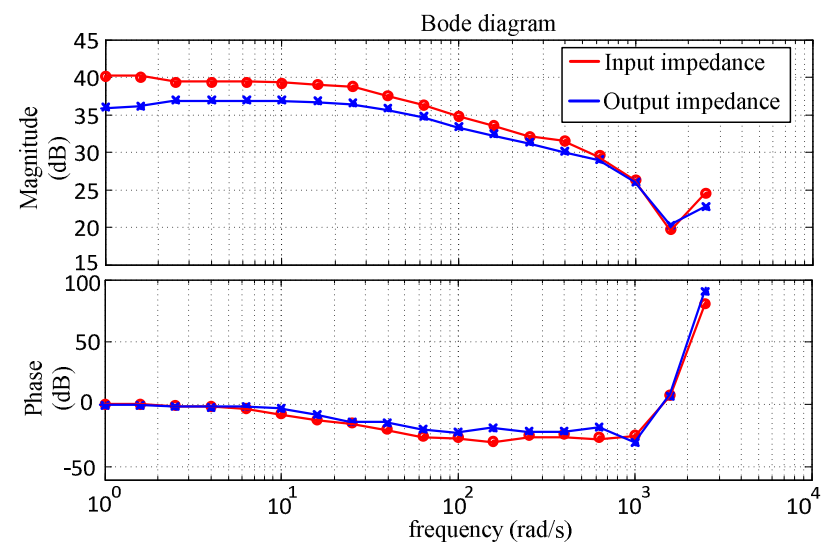

Fig. 16 Impedance measurement of the proposed control

To compare the stability differences, the output impedance of DAB converter is intentionally increased, then the system stability will be deteriorated, as shown in Fig. 15. In the simulation, the power reference is a periodic square wave, with the period of $1 \mathrm{~s}$, changing between $-5000 \mathrm{~kW}$ and $5000 \mathrm{~kW}$. Fig. 17 shows the simulation results. Before $1.4 \mathrm{~s}$, it is the conventional 


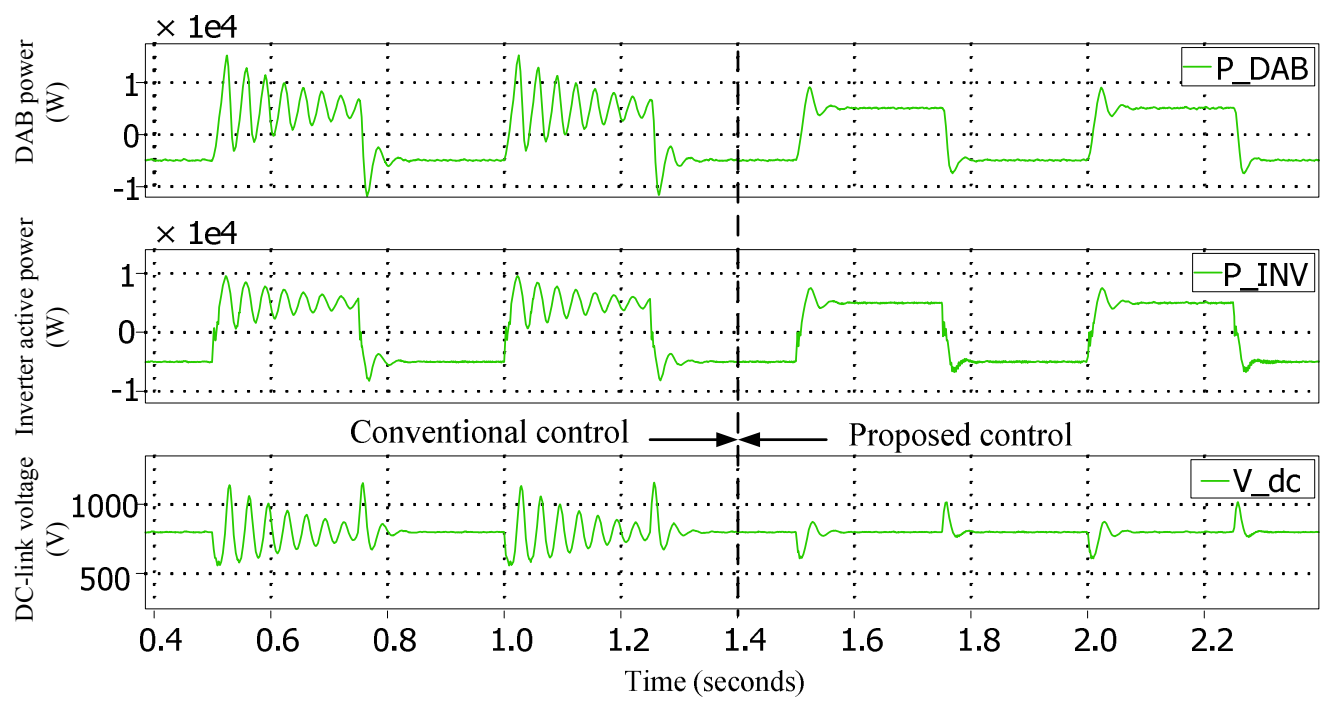

Fig. 17 Simulation results of conventional control and the proposed control

control, and after $1.4 \mathrm{~s}$ is the proposed control. As shown, in conventional control, the oscillations in forward power flow are more severe than in the reversed power flow, and it indicates the stability in reversed power flow is better than that in the forward

flow. While in the proposed control, under the same condition, the oscillation in forward power flow has much been greatly damped in the forward power flow, and the dynamic performance of forward power flow is almost the same as the reversed power flow. So the stability in the proposed control is almost the same between forward power flow and reversed power flow.

\section{B. Experiment results}

A scaled down prototype has been built. In the experiment, the power reference is the periodic square waves, between $400 \mathrm{~W}$ and $-400 \mathrm{~W}$, with the period of $2 \mathrm{~s}$.

Fig. 18 shows the experiment results. Before the dash line, the control is conventional control, and after dash line, the proposed control is activated.

As shown in Fig. 18, in conventional control, the dynamic oscillation in forward power flow is more server than in the reversed power flow. While in the proposed control, the oscillations become smaller and similar between forward and reversed power flow. It can be seen that the proposed control can improve the stability in forward power flow, and makes the system have with similar stability between forward power flow and reversed power flow.

Fig. 19 depicts the waveforms of DC-link voltage, inverter power and current output. As shown in Fig. 19 (a), in conventional control, inverter current and power has small oscillations when the power output is $400 \mathrm{~W}$. While in Fig. 19 (b), the proposed control, the steady state of power output is much better.

Fig. 20 shows the experiment results of DC-link voltage and $\mathrm{DAB}$ power, current output. As shown, in conventional control, there is significant oscillation in DAB current and power output, while in the proposed control, the waves forms become much stable.

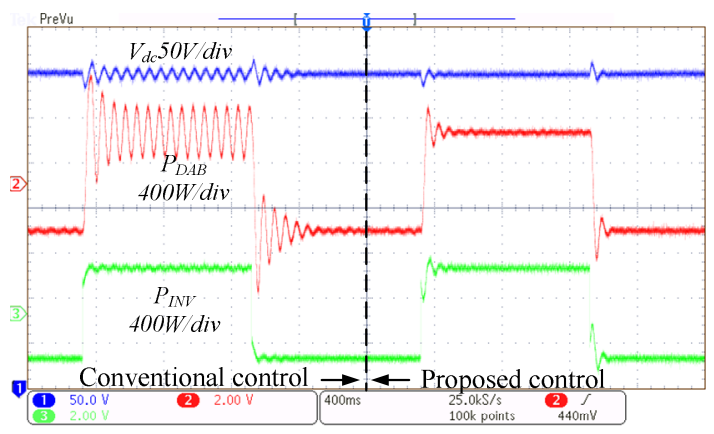

Fig. 18 Experiment results of the DC-link voltage and DAB inverter power output

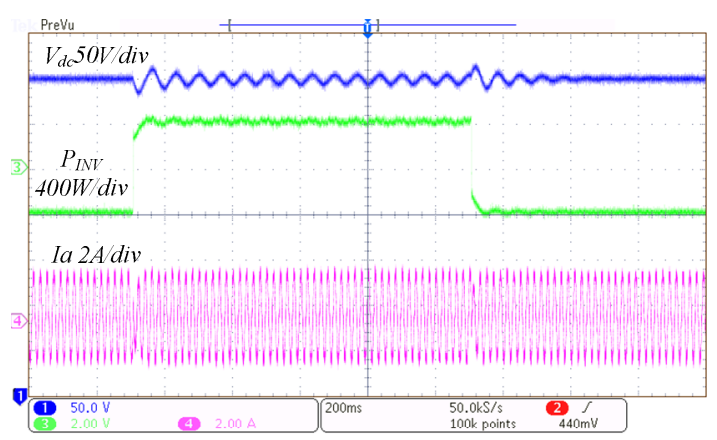

(a) Conventional control

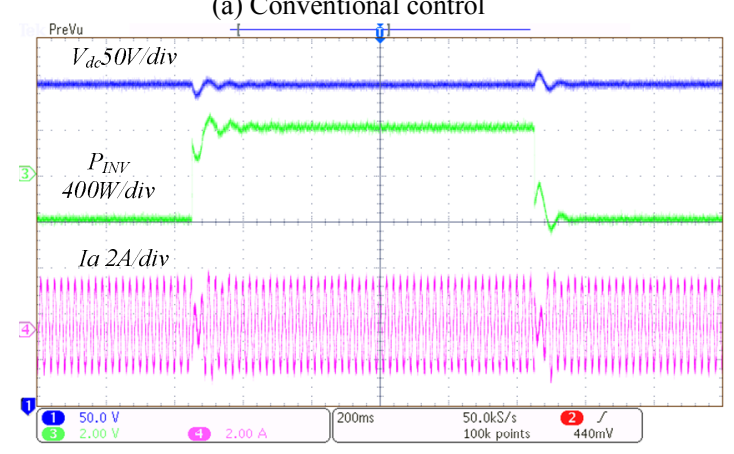

(b) The proposed control

Fig. 19 Experiment results of the DC-link voltage, inverter power and current output 


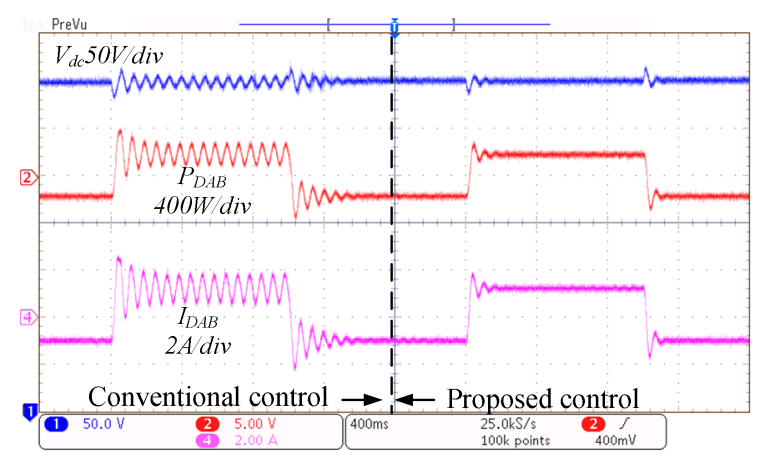

Fig. 20 Experiment results of the DC-link voltage, DAB power and current output

\section{CONCLUSION}

With the existence of CPLs converter, cascaded system stability in forward power flow is worse than the reversed power flow. This paper proposed an impedance coordinate control for the two stages cascaded converter. The proposed control can improve the stability in forward power flow, and make the impedance interaction behave more similar between forward and reversed power flow. The impedance modelling has been offered, as well as the bode plots and nyquist plots. In the proposed control, the constant power controlling converter behaves as resistive impedance, and behaves more unity in different power flow direction. The proposed control can also be implemented in the cascaded converter system with other topolgoy. The conclusion has been validated by both the simulation and experimental results.

\section{ACKNOWLEDGMENT}

The authors would like to thank the Danish Council for Strategic Research for providing the financial support under the project "Development of a Secure, Economic and Environmentally-friendly Modern Power Systems" (DSF 09-067255.)

\section{REFERENCES}

[1] Frede Blaabjerg, Zhe Chen, Soeren Baekhoej Kjaer, " Power Electronics as Efficient Interface in Dispersed Power Generation Systems," IEEE TRANSACTIONS ON POWER ELECTRONICS, vol. 19, NO. 5, SEPTEMBER 2004

[2] Juan Manuel Carrasco, Leopoldo Garcia Franquelo, Jan T. Bialasiewicz, Eduardo Galván, Ramón C. Portillo Guisado, Ma. Ángeles Martín Prats, José Ignacio León, Narciso MorenoAlfonso, :" Power-Electronic Systems for the Grid Integration of Renewable Energy Sources: A Survey," IEEE Transactions on Industrial Electronics, vol. 43, NO. 4, August 2006.

[3] Kai Zenger, Ali Altowati, Teuvo Suntio "Dynamic Properties of Interconnected Power Systems - A System Theoretic Approach," ICIEA 2006.

[4] R.D. Middlebrook,"Input filter considerations in design and application of switching regulators, " in Proc. IEEE IAS,1979, pp 366-382.

[5] S. Vesti, J.A. Oliver, R. Prieto, J.A. Cobos, T. Suntio, "Stability and Transient Performance Assessment in a COTS-Module-Based Distributed DC/DC System," Telecommunications Energy Conference (INTELEC), 2011 IEEE 33rd International, 9-13 Oct. 2011, Amsterdam, 2011, Page(s): 1 - 7.
[6] Antonino Riccobono, and Enrico Santi, "Positive Feedforward Control of Three-Phase Voltage Source Inverter for DC Input Bus Stabilization With Experimental Validation," IEEE TRANSACTIONS ON INDUSTRY APPLICATIONS, vol. 49, NO. 1, JANUARY/FEBRUARY 2013.

[7] Ali Emadi, Alireza Khaligh, Claudio H. Rivetta, Geoffrey A. Williamson, "Constant Power Loads and Negative Impedance Instability in Automotive Systems: Definition, Modeling, Stability, and Control of Power Electronic Converters and Motor Drives," IEEE TRANSACTIONS ON VEHICULAR TECHNOLOGY, VOL. 55, NO. 4, JULY 2006.

[8] Amir M. Rahimi, Ali Emadi, "Active Damping in DC/DC Power Electronic Converters: A Novel Method to Overcome the Problems of Constant Power Loads," IEEE TRANSACTIONS ON INDUSTRIAL ELECTRONICS, VOL. 56, NO. 5, MAY 2009.

[9] Jiabin Wang, David Howe, "A Power Shaping Stabilizing Control Strategy for DC Power Systems With Constant Power Loads," IEEE Transactions on Power Electronics, Vol. 23, No. 6, November.

[10] Hua Bai, Chris Mi, "Eliminate Reactive Power and Increase System Efficiency of Isolated Bidirectional Dual-Active-Bridge DC-DC Converters Using Novel Dual-Phase-Shift Control," IEEE Transactions on Power Electronics, VOL. 23, NO. 6, NOVEMBER 2008

[11] Xiaogang Feng, Zhihong Ye, Kun Xing, Fred C. Lee, Dusan Borojevic," Individual Load Impedance Specification for a Stable DC Distributed Power System," Applied Power Electronics Conference and Exposition, 1999. APEC '99. Fourteenth Annual, 14-18 Mar, Dallas, USA, 1999, PP 923 - 929.

[12] S. Vesti, T. Suntio, J. A. Oliver, R. Prieto, J. A. Cobos, "Impedance-Based Stability and Transient Performance Assessment Applying Maximum Peak Criteria," IEEE TRANS ON POWER ELECTRONICS, vol. 28, NO. 5, MAY 2013.

[13] Carl M. Wildrick, Fred C. Lee, Bo H. Cho, Byungcho Choi, "A Method of Defining the Load Impedance Specification for A Stable Distributed Power System," IEEE Transactions on Power Electronics, Vol. 10, NO. 3. May, 1995.

[14] J. Lepp"aaho, J. Huusari, L. Nousiainen, J. Puukko, and T. Suntio, "Dynamic properties and stability assessment of current-fed converters in photovoltaic applications," IEEJ Trans. Ind. Appl., vol. 131, no. 8, pp. 976-984, Aug. 2011.

[15] Xing Zhang, Chongwei Zhang, "PWM rectifier and control," Machinery Industry Press, October, 2003, pp.148-153.

[16] Xin Zhang, Xinbo Ruan, Hyok Kim, Chi K.Tse, "Adaptive Active Capacitor Converter for Improving Stability of Cascaded DC Power Supply System ," IEEE Transactions on Power Electronics, Vol. 28, No. 4, April 2013.

[17] Zengshi Chen, Wenzhong Gao, Jiangang Hu, Xiao Ye, "ClosedLoop Analysis and Cascade Control of a Nonminimum Phase Boost Converter," IEEE TRANSACTIONS ON POWER ELECTRONICS, VOL. 26, NO. 4, APRIL 2011.

[18] Hany M. Hasanien, S. M. Muyeen, "Design Optimization of Controller Parameters Used in Variable Speed Wind Energy Conversion System by Genetic Algorithms," IEEE TRANSACTIONS ON SUSTAINABLE ENERGY, VOL. 3, NO. 2, APRIL 2012.

[19] Jianjiang Shi, Wei Gou, Hao Yuan, Tiefu Zhao, Alex Q. Huang, "Research on Voltage and Power Balance Control for Cascaded Modular Solid-State Transformer," IEEE TRANSACTIONS ON POWER ELECTRONICS, VOL. 26, NO. 4, APRIL 2011.

[20] Harish K. Krishnamurthy, Raja Ayyanar, "Building Block Converter Module for Universal (AC-DC, DC-AC, DC-DC) Fully Modular Power Conversion Architecture,"

[21] Remus Teodorescu, Marco Liserre, Pedro Rodr'́ruez, Grid Converters for Photovoltaic and Wind Power, 1st edition, A John Wiley and Sons, Ltd., Publication, pp. 222.

[22]Yanjun Tian, Fujin Deng, Zhe Chen, Xiaofeng Sun, Yanting Hu, "Impedance Interaction modeling and Analysis for the Bidirectional Cascaded Converter,"Accepted in ICPE 2015ECCE, Seoul, Korea. 